\title{
Atención integral a los problemas de convivencia familiar, en una comisaría de familia de la ciudad de Cali*
}

\section{Integral attention to family issues in a family comissioner's office in Cali, Colombia}

\author{
Adriana Mora Anto* \\ Miriam Román Muñoz \\ Valentina Varela Martínez \\ Pontificia Universidad Javeriana, Cali, \\ Colombia
}

Recibido: 5 de junio de 2014 Revisado: 13 de agosto de 2014 Aceptado: 1 de octubre de 2014

\section{Resumen}

En esta investigación cualitativa se abordaron los procesos de atención integral a los problemas de convivencia familiar, en la Comisaria de Familia del sector Ladera en Cali, teniendo en cuenta la perspectiva de los modelos alternativos de resolución del conflicto. La Comisaría cuenta con un protocolo definido para la atención de los casos, además de una ruta de atención. De acuerdo con los Informes Anuales de Labores, en el período comprendido entre 2007 y 2011, se registró la atención de 39.189 personas, siendo más frecuentes las quejas por violencia familiar; adicionalmente, se reciben casos de alimentos, custodias y visitas de menores. La Comisaria prescribe medidas conciliatorias y de protección provisional con las que busca velar por la convivencia familiar.

Palabras clave: convivencia familiar, atención integral, solución de conflictos.

Correspondencia: Adriana Mora Anto, Grupo de Investigación Estudios en Cultura, Niñez y Familia. Departamento de Ciencias Sociales, edificio Edificio El Samán Oficina 3.46. Pontificia Universidad Javeriana Cali. Este artículo es producto de la investigación “Convivencia Familiar en Cali: Atención a los casos de violencia familiar en Comisarías de Familia, 2005 - 2011" realizada durante el año 2012, financiada por la Pontificia Universidad Javeriana Cali y registrada bajo el código 020100302 de la Oficina General de Investigaciones.

Agradecimientos a la Subsecretaría de Policía y Justicia de la Alcaldía de Santiago de Cali y a los funcionarios de la Comisaría de Familia del sector Ladera que participaron en esta investigación. 


\section{Abstract}

This qualitative research addressed the processes of integral attention to the problems of family life, in the Family Commissioner in Cali, taking into account the perspective of alternative conflict resolution models. The Commissioner has a defined protocol for the attention of cases, in addition to a route of attention. According to annual reports of work, in the period between 2007 and 2011, the care of 39.189 people registered, being most frequent complaints it by family violence; in addition, cases of food, custody and visits by minors are received. The Commissioner prescribes conciliatory and interim protective measures which seeks to ensure the family life.

Keywords: Family coexistence, integral attention, conflict resolution

\section{Introducción}

En la vida de cualquier persona, la familia siempre emerge como un elemento fundante de su subjetividad, independiente de lo acogedora y nutricia que haya sido, o de la funcionalidad que haya demostrado para ajustarse a las demandas y a los cambios que le plantean sus integrantes o los que propone el entorno social y cultural. Los contextos culturales, las costumbres, las interacciones cada día son más diversas, y representan una complejidad mayor para el estudio de las familias y de sus integrantes.

Ackerman (citado en Eguiluz, 2003) explica que la familia tiene como función prioritaria la conservación de la especie; además, promueve las interacciones afectivas y crea los vínculos de interdependencia necesarios para la satisfacción de las necesidades de sus integrantes, las habilidades para el crecimiento personal, la diferenciación de labores y la previsión de las necesidades materiales. La familia como espacio de socialización aporta su cuota a la formación de seres humanos pensantes y participativos, que puedan sumarse a la convivencia social y al fortalecimiento de la ciudadanía. De igual manera, su idealización también comporta el riesgo de estigmatizarla como la única instancia responsable de las dificultades relacionales, e incluso violentas, que experimentan las personas.

En este sentido, es necesario aclarar que la violencia familiar es producto de una construcción social en la que intervienen una diversidad de factores, y su estudio implica el análisis de los condicionantes culturales, sociales, económicos y políticos, lo que muestra la necesidad de profundizar en la forma como se promueve la convivencia familiar, tanto desde la normatividad jurídica como en la aplicación de ella.

De acuerdo con los informes del Observatorio de Violencia Familiar (OVF) de la Secretaría de Salud de la ciudad de Cali, se registra una tendencia creciente en la denuncia de delitos por violencia familiar. En su mayoría, los casos de violencia familiar son reportados en las Comisarías de Familia del sector Ladera Oeste y Oriente, dos de las zonas más conocidas por sus precarias condiciones económicas y alta densidad poblacional.

Según el Informe del OVF (2008), el 52\% de las personas que fueron víctimas de violencia familiar no cuentan con un sistema de seguridad social. Las mujeres fueron las más afectadas por violencia familiar de todo tipo, y en todos los grupos de edad; el tipo de maltrato más frecuente es el psicológico, así como la negligencia y el abandono. Los agresores reportados con mayor frecuencia son la madre y el padre ( $40 \%$ de los casos); el cónyuge $(20,3 \%)$ y el ex cónyuge (12,6\%).

Una nota adicional es que en la zona oriente de la ciudad reside más de la tercera parte de los casos reportados, y en el sector de Ladera se ubica la cuarte parte, lo que equivale al $60,3 \%$ de los casos reportados. En 2012, las cifras de violencia 
familiar reportadas por el OVF refieren 6453 casos, siendo las mujeres entre 20 y 24 , y entre 30 y 34 años de edad las principales víctimas. Esto significa que la atención a los problemas de convivencia familiar sigue siendo urgente y sugiere la necesidad de prestar más atención a la integralidad de la intervención por parte de las entidades responsables.

En Colombia, la creación de las comisarías de familia surge como una respuesta del Estado a su obligación de garantizar la protección de la familia como núcleo fundamental de la sociedad (Decreto 2737 de 1989). Las comisarías de familia son entidades de carácter administrativo e interdisciplinario, cuya misión es prevenir, garantizar, restablecer y reparar los derechos de los miembros de la familia que han sido violentados por diversas situaciones que establece la ley colombiana, lo que significa que la atención que se brinda a los problemas de convivencia familiar es responsabilidad directa de las comisarías.

En la constitución colombiana, la familia se define como "el núcleo fundamental de la sociedad. Se constituye por vínculos naturales o jurídicos, por la decisión libre de un hombre y una mujer de contraer matrimonio o por la voluntad responsable de conformarla" (Artículo 42, CPC, 1991). Sin embargo, Guío (2009) aclara que este concepto en la legislación colombiana también se extiende a otras formas de grupos familiares, por ejemplo, cuando se refiere a la mujer cabeza de hogar que ha sido desplazada por causa del conflicto armado; a los niños y su derecho a tener una familia y no ser separados de ella; a la necesidad de una edad mínima para adoptar; y a la protección a las parejas del mismo sexo en el ámbito patrimonial (p. 66), a lo que se suma la Ley 1361 de 2009 cuando alude a la atención integral e integración social de las familias bajo un enfoque de derechos de equidad, solidaridad y participación familiar, brindando atención integral a las familias en situación especial de riesgo.

En consecuencia, las comisarías tienen la responsabilidad de "recibir y tramitar las solicitudes de protección que formulen los ciudadanos o ciudadanas por hechos de violencia intrafamiliar", según la Ley 294 de 1996, Ley 575 de 2000, Ley
1257 de 2008 y Ley 1098 de 2006. Además, tienen autoridad con facultades conciliatorias, referida a conciliaciones extrajudiciales en materia de familia, en asuntos susceptibles de conciliación (según la ley vigente) y a cuestiones como desacuerdos sobre custodias y el régimen de visitas sobre menores e incapaces; asuntos relacionados con obligaciones alimentarias; declaración de unión marital de hecho, su disolución y la liquidación de la sociedad patrimonial; anulación de procesos por sucesiones y liquidación de la sociedad conyugal; conflictos sobre capitulaciones matrimoniales; separación de bienes y cuerpos; y controversias entre cónyuges sobre la dirección conjunta del hogar, y entre padres sobre el ejercicio de la autoridad paterna o la patria potestad, además de la fijación de la cuota alimentaria.

En estos casos, la ley entiende por violencia intrafamiliar "toda forma de maltrato físico, verbal o atropello a las esferas sexual, psíquica, emocional y afectiva de la víctima" (artículo 1 de la Ley 575 de 2000). La violencia intrafamiliar se considera un delito querellable, es decir, un delito para el cual es necesario iniciar una acción penal ante la Fiscalía y observar procedimientos de denuncia establecidos en la ley. Sin embargo, muchas de las quejas o reclamos por situaciones asociadas a la violencia intrafamiliar, o que representan un riesgo para su ocurrencia, se formulan ante la Comisaría de Familia o son remitidas a ella: en muchas ocasiones, los usuarios (generalmente, las mujeres) acuden a la comisaría con la intención de que se haga un llamado de atención a la pareja o al padre maltratador que incumple con la obligación alimentaria, antes de que se le fije una medida de arresto o reclusión.

Es en estas situaciones que las comisarías y los funcionarios tienen un papel central como promotores de la convivencia familiar, de forma que contribuyan a redimensionar las relaciones en los grupos familiares, e incluso a convertirse en una oportunidad para aprender a negociar las reglas de la convivencia. Desde la perspectiva psicológica, es importante comprender que la violencia familiar surge allí donde los integrantes del grupo familiar no encuentran alternativas que les permitan resolver sus desacuerdos en materia de 
conflicto (Barudy, 1998; Bentovim, 2000), lo cual tiende a complejizarse al punto de transformarse en un patrón violento de relación. Se trata de una contradicción en la que los miembros de la familia o parientes se relacionan desde polos opuestos, desde necesidades diferentes, pero con fines comunes: mantener el control y, quizás también, preservar una relación que se teme perder.

Barudy (1998) define la violencia como un problema relacional complejo, multicausal y multicondicional, que requiere un modelo ecosistémico que permita, en la intervención, entender y complejizar, atender y solucionar de forma integral, interdisciplinaria e intersectorial los problemas que se presentan en la convivencia familia; señala que tanto las personas abusadoras, como las abusadas y terceros, son actores involucrados. Por lo tanto, el cambio al que se debe llegar es en el estilo de las relaciones abusivas, en la estructura relacional, razón por la cual en las acciones deben participar los tres actores responsables (Pineda y Otero, 2004).

En este marco de ideas, esta investigación atendió a los procesos de atención integral a las quejas por problemas de convivencia familiar y al restablecimiento de los derechos en una comisaría de familia de la ciudad de Cali, en los que se demandaba la intervención de los funcionarios públicos en calidad de terceras personas competentes que representasen al Estado para promover la convivencia. Esta investigación se enfocó en la necesidad de analizar el papel que los funcionarios de las comisarías de familia tienen en el mejoramiento y garantía de una mejor convivencia familiar y ciudadana.

Al respecto, la Política pública en convivencia familiar para el municipio de Cali (2005) señala que

la tensión entre la definición de familia de la normatividad y la vida cotidiana obliga a señalar los obstáculos jurídicos para avanzar en legislaciones más incluyentes de familia y violencia familiar [...] la violencia familiar es reconocida como un problema social y desde ahí hay que resaltar sus avances, pero aún falta para que el fenómeno sea atendido con la calidad y responsabilidad por las instituciones que tienen a cargo ese hacer. La realidad desborda la capacidad institucional y la calidad de la atención depende también de los recursos y del número de funcionarios; se requiere de profundizar en el conocimiento de este fenómeno a través de la investigación (pp. 32-33).

De igual forma, a propósito de la convivencia familiar, el Mandato Ciudadano señala que "La convivencia familiar es un asunto de carácter público y le compete al Estado responsabilizarse de manera conjunta con la sociedad civil" (Alcaldía de Santiago de Cali, Red del Buen Trato, Fundación para la Orientación Familiar [FUNOF] y Foro Familia y Convivencia, 2004, p 172). A los señalamientos de este informe, pueden agregarse las recomendaciones del Informe de Medición del Cultura Ciudadana 2013, cuando menciona que es necesaria una atención más integral para los casos de violencia familiar que se enfoque también en la educación e intervención psicoterapéutica con las victimas y los victimarios.

Estudios como el de Victoria y Bonilla (2008) acerca de los imaginarios sobre los funcionarios de justicia que atienden los casos de violencia sexual enfatizan en el riesgo de la revictimización cuando se escucha la demanda de las mujeres que han sido abusadas sexualmente, sin considerar las incidencias que la forma de intervenir y cuestionar del funcionario puedan tener sobre las emociones de la mujer abusada. En otro sentido, estudios como el de Rentería, Lledias y Giraldo (2008) muestran la influencia favorable que las redes de apoyo comunitarias y la vinculación de las personas con instituciones que las educan en el conocimiento de sus deberes y sus derechos tienen en el trato a los hijos.

\section{Objetivos}

\section{Objetivo general}

Analizar los procesos de atención integral a los conflictos de convivencia familiar en una comisaría de familia de la ciudad de Cali y su contribución a la promoción de la convivencia familiar. 


\section{Objetivos específicos}

- Comprender el tipo de procedimientos de atención que los funcionarios realizan al recibir denuncias sobre conflictos de convivencia familiar.

- Establecer la intencionalidad que subyace a los procedimientos de atención integral de los funcionarios, en términos de la resolución del conflicto y la promoción de la convivencia familiar y social.

\section{Metodología}

Esta investigación se basó en el análisis de los informes anuales de gestión (2007-2012) de la Comisaría de Familia del sector Ladera, que cobija a las comunas 1, 3, 17, 18, 19, 20 y 22 en la ciudad de Cali, y en la que se registran el $25,5 \%$ de los casos de violencia familiar, reportados por el Informe del Observatorio de Violencia Intrafamiliar (OVI, 2008) de la Secretaría de Salud Municipal.

Además, recogió información cualitativa derivada de las entrevistas con el grupo de profesionales de diferentes disciplinas de la comisaría, la realización de un grupo de discusión con los funcionarios, junto con las observaciones de los procesos de atención a usuarios (procesos de registro, audiencias y taller educativo con usuarios), a los que sumaron la revisión de las leyes 294 de 1996, 575 de 2000 y 1257 de 2008 (entre otras), el $L i$ neamiento técnico para comisarías de familia del Instituto Colombiano de Bienestar Familiar y los informes anuales de gestión de la comisaría, contando con el permiso necesario para el desarrollo del estudio.

\section{Resultados}

El lineamiento técnico del Instituto Colombiano de Bienestar Familiar establece que las comisarías estarán conformadas como mínimo por un abogado -quien asumirá la función de comisario(a)-, un psicólogo(a), un trabajador(a) social, un(a) médico(a) y un(a) secretario(a), que trabajaran en equipo. También podrán apoyarse en otros profesionales que trabajen sobre el tema de familia, de forma que garanticen la protección de los derechos de los niños y sus padres.

El equipo de trabajo de la comisaría de familia en la que se realizó la investigación está compuesto por profesionales de Derecho, Psicología y Trabajo Social, además de profesionales de carrera administrativa, quienes, en su mayoría, tienen un contrato por nombramiento. Sin embargo, cuando el profesional de apoyo no tiene un contrato por nombramiento, desempeña un cargo como contratista temporal, que asume sus funciones por periodos de tiempo definidos y no permanentes, lo que dificulta los procesos de atención de forma más oportuna.

Una contextualización de las cifras de atención en la comisaría elegida indica que en el periodo 2007-2012 se expidieron un total de 42.471 boletas de citación y se atendieron 39.189 personas. De acuerdo con los registros del Informe Anual de Labores (2007-2012), se prestaron 29.818 asesorías legales, a las que se agregan 3232 asesorías psicológicas y 1354 orientaciones de trabajo social (Comisaría de Familia, sector Ladera, Cali, 2012).

De acuerdo con los procedimientos de atención, toda víctima de violencia familiar puede solicitar una medida de protección en un plazo no inferior a los treinta días, después de sucedido el hecho de agresión; el comisario ordenará al agresor abstenerse de realizar la conducta u otros actos de violencia en contra de los integrantes de la familia y recurrirá a las medidas necesarias para garantizarlo.

En cuanto a las medidas de protección, el comisario recibirá denuncias y adaptará las medidas necesarias en los casos de delitos contra niños, niñas y adolescentes, además de orientarlos a ellos y sus familias en el ejercicio y restablecimiento de sus derechos, e intervenir en situaciones de urgencia en las que se les maltrate y violente. En lo referente a medidas de prevención, le corresponde el desarrollo de programas en materia de violencia intrafamiliar y delitos sexuales.

Un aspecto importante de las competencias de una comisaría de familia se refiere al criterio que diferencia sus funciones de una defensoría de fa- 
milia: la presencia o no de violencia familiar en la situación que origina la queja o la denuncia, por tratarse de vulneración de los derechos de los niños, niñas y adolescentes. Por ello, corresponde a la comisaría ocuparse de las situaciones de violencia intrafamiliar, si el evento de maltrato, amenaza o vulneración de los derechos ocurre en el contexto de violencia intrafamiliar. Estos constituyen los principios generales sobre los que se justifica la labor de las comisarías de familia y su papel en la promoción de la convivencia familiar.

La comisaría de familia atiende quejas en relación con la convivencia familiar en lugar de atender las denuncias, ya que de acuerdo con el Decreto 4840 de 2007, su función es contribuir a la resolución de conflictos asociados a la violencia familiar, enfocándose en la prevención, la sanción y la reparación, funciones que se desarrollan con el apoyo de otras instituciones como la Policía, las instituciones prestadoras de servicios de Salud (IPS) y la Fiscalía, por lo que la reparación se convierte en un elemento mediador que busca contener la violencia en la familia, y que tiene una influencia decisiva en la forma como las personas afrontan el conflicto y dan fin a sus diferencias.

De acuerdo con las observaciones realizadas, la labor del funcionario como contenedor de la violencia incide en el cese de la reacción violenta inmediata, a pesar de que los motivos de los desacuerdos que originaron el conflicto no necesariamente se hayan resuelto, o se hayan encontrado formas de relación alternativa.

La atención se complementa con la orientación psicoeducativa que adelantan los profesionales del campo psicosocial, junto con las IPS a las que son remitidos los usuarios y las medidas de protección que prescribe la comisaría con el apoyo de la Policía. Incluso la asistencia a los talleres psicoeducativos es obligatoria para todos aquellos individuos que hayan formulado quejas por situaciones que hayan conducido o puedan conducir a la violencia familiar. Otros recursos para la prevención de la violencia familiar se refieren a las acciones de seguimiento, como las visitas domiciliarias y jornadas de trabajo con la comunidad, que se programan en la zona de intervención de la comisaría.
El proceso de atención tiene varias etapas: la primera es la conciliatoria, que se realiza cuando se han agotado los recursos de solución del conflicto entre los sujetos procesales, y ellos recurren a la audiencia con la comisaría. En esta etapa, se advierte a las partes sobre lo negativo e inconveniente de su conducta, y se los exhorta a cambiar de actitud; además, se prescriben las medidas de protección como la orientación psicológica, orientación familiar, protección de la Policía, asistencia a un hogar de paso, e incluso una visita domiciliaria.

No obstante, es posible que en esta etapa de conminación inicial (o también conciliatoria) se formule una caución contra una de las partes o ambas partes, que equivale al establecimiento de una multa (entre dos y diez salarios mínimos legales mensuales, que deberán consignarse dentro de los cinco días siguientes a su imposición [literal a del artículo 4 de la Ley 575 de 2000]) o la medida de arresto (45 días), que se hará efectivo cuando una de las partes o ambas denuncien haber sido objeto de violencia familiar.

La segunda etapa corresponde a la existencia de incidentes, en la que se trata de probar que existió un incumplimiento de la medida de protección que se dictó en la audiencia. En este sentido, con la finalidad de asegurar y ofrecer garantía de derechos a las personas, se decreta un periodo probatorio para demostrar que ha existido el incumplimiento de la medida, por lo que es necesario que las personas aporten las pruebas y la evidencia necesaria. La tercera etapa conciliatoria, tiene resultados muy positivos, porque consigue que la acción violenta se suspenda.

En la comisaria de familia analizada, de 13.000 casos solamente un caso ha llegado a tercera etapa, de acuerdo con el reporte administrativo. En lo que se refiere al seguimiento y la posibilidad de reincidencia en la conducta de violencia, la ley permite seguir el caso hasta dos años después de presentada la denuncia. Sin embargo, de acuerdo con el artículo 4 de la Ley 575, literal a, no es posible inmiscuirse en la intimidad de las familias, puesto que se respeta el derecho a la privacidad; solo es posible hacer este seguimiento cuando se 
demuestra la existencia de conductas violentas entre las personas. De ahí la necesidad de aportar pruebas para la justificación de un incidente de desacato a las medidas iniciales, aunque, como ya se dijo, los casos de reincidencia no son frecuentes en los registros de la comisaría.

El mayor número de casos que se reciben en la comisaría son por violencia familiar, violencia contra la mujer y violencia contra los niños, aunque no se descarta la violencia contra los hombres. En septiembre de 2012, por ejemplo, el informe anual de gestión muestra que se registraron: 292 cauciones, 32 conciliaciones, 3 multas, 200 asesorías de trabajo social y 431 orientaciones psicológicas, asociadas a asuntos de convivencia y maltrato (por ejemplo, atención de casos de alimentos, custodias y visitas de menores [Comisaría de Familia, 2012]).

Este alto número de quejas se atribuye a la desinformación de la comunidad en cuanto a las competencias de la comisaría y otras entidades públicas, como los centros zonales del ICBF y la Fiscalía. Adicionalmente, los escenarios en los que se manifiestan los problemas de violencia familiar casi siempre son los hogares, rodeados de problemas de pareja como los celos, la infidelidad y el consumo de drogas. Los casos de mayor severidad son aquellos en los que han existido amenazas de muerte, en razón a la inminencia del riesgo para las víctimas.

Un aspecto fundamental es que, de acuerdo con los lineamientos del Sistema Nacional de Bienestar Familiar, se requiere la adopción del modelo solidario de intervención con familias, en el que la unidad de análisis es la familia o las redes vinculares, sociales e institucionales en las que se inscribe la persona. La adopción de este modelo implica que el equipo profesional de la comisaría, en conjunto con la familia, identifiquen los recursos, logros y potencialidades de aquella, o trabajen para construirlos, de modo que el profesional interventor se convierta en un "acompañante, cuya mayor fortaleza es la de visibilizar y activar los recursos de la familia, los individuos que la integran y las redes", lo que lo convierte en un facilitador del cambio, que analiza y define la de- manda de atención junto con la familia, y construye soluciones en forma colaborativa, apoyándose en la red de relaciones familiares e institucionales (ICBF, 2008, p. 38).

Se advierte que el modelo de intervención solidario valora la identificación de las tareas, metas y desafíos propios de la etapa del ciclo vital en que se ubica la familia, al igual que la participación e inclusión de aquella en la generación del cambio, es decir, se propone evitar la creación de una actitud de carácter asistencialista y, en lugar de ello, promover la capacidad para resolver conflictos y aprender a convivir.

De alguna forma, puede decirse que se busca que el espacio jurídico represente también un espacio de alivio psíquico para quien formula la queja, pues se trata de que detrás de la queja exista también la necesidad de recuperar un estado emocional anterior a la situación que motiva la queja. Además, es claro que las parejas que asisten motivadas por conflictos en relación con la custodia de los hijos o problemas de agresión, también buscan resolver conflictos por la manutención, la custodia o el maltrato que reciben los niños.

Frente a esta situación, los funcionarios consideran que existe la necesidad de un seguimiento más allá de lo legal, a través de las visitas domiciliarias y la intervención con el núcleo familiar para la generación de cambios en las relaciones; sin embargo, los profesionales en el área psicosocial son pocos, y trabajan como asesores y orientadores, mas no como interventores permanentes. Otro elemento que dificulta la labor es la existencia de contrataciones temporales por razones presupuestales.

Finalmente, anotan que la comisaría tiene visitas de inspección y vigilancia por parte de la Personería, que es la instancia de control y vigilancia en cuanto a los derechos humanos y constitucionales; además, existe el mecanismo jurídico de las tutelas, al que pueden apelar los usuarios cuando quieren reclamar la nulidad de las medidas de protección, recurso al que han recurrido algunas pocas personas de estrato alto. 
De acuerdo con el equipo de trabajo de la comisaría de familia que se entrevistó, esta dependencia es considerada como un modelo de organización y eficiencia en el proceso de atención, en el sentido de contar con un sistema de información organizado, completo y actualizado, además de presentar reportes de información completos a la Subsecretaría de Policía y Justicia, dependencia municipal de la que depende, y ante la Alcaldía Municipal.

\section{Discusión de resultados}

Los procesos de atención en la comisaría de familia se ordenan dentro de lo prescrito por la Ley 575 de 2000 sobre asuntos de familia, la Ley 1098 respecto a infracciones de menores y el Decreto 441 de 1998 para la descongestión de la administración de justicia. Estas leyes actúan como marco para la intervención de los funcionarios, quienes interpretan la administración de justicia en términos de la "mitigación" de la violencia familiar, pues la comisaría de familia tiene como misión

prevenir, orientar, mitigar, sancionar y tratar los problemas pertinentes a las familias, con el propósito de restablecer la armonía en el hogar, promover la convivencia y prevenir la violencia familiar y garantizar los derechos de todos los ciudadanos y los miembros de las familias.

No obstante, existe una distinción fundamental en torno a la intervención jurídica y psicosocial de la siguiente forma:

1. La protección se ejecuta a través de las medidas conformes con la ley.

2. La sanción se establece mediante la fijación de las cauciones a las personas implicadas en hechos de violencia familiar que, durante las audiencias, se muestren reacias o persistan en maltrato y agresiones verbales. En este caso, se insta a las personas para asistir a los talleres educativos y al servicio de psicología o trabajo social, a quienes corresponde la intervención psicosocial.

3. La protección y la reparación se logra a través de las redes de apoyo que son dependencias estatales como la Policía, la Fiscalía, los hoga- res de paso, el Instituto de Medicina Legal y las instituciones prestadoras de servicios en salud, además del servicio de psicología y trabajo social de la comisaría. También colaboran las universidades, a través de los consultorios jurídicos y las instituciones educativas con el tema de prevención.

Estas distinciones marcan limites respecto a cómo se interviene frente a la violencia familiar y se resuelven los conflictos de la convivencia, dado que la resolución se funda en el análisis de las quejas de cada una de las personas involucradas y en la prescripción de una multa que provoca el cese casi inmediato de las acciones violentas.

Este tipo de intervención es de estricta competencia jurídica y responde al seguimiento de la norma jurídica. Sin embargo, enfatiza en el seguimiento de las normas, más que en la resolución del conflicto y la construcción de convivencia, aunque el interés particular de los profesionales se oriente hacia la construcción de aquella.

Esta intervención se limita por varias razones: la escogencia de una intervención basada en la norma jurídica, por considerar que tiene un efecto inmediato que la hace eficiente; y la carencia de un sistema de medición del impacto de la intervención de los funcionarios, que sea congruente con los propósitos para los que fue creada la comisaría de familia, como entidad estatal responsable de la descongestión del sistema judicial y la promoción de la convivencia familiar, además del tipo de contratación temporal que tienen algunos funcionarios, dado que limita su tiempo para la intervención.

En la intervención conciliatoria se recurre al establecimiento de una multa, cuando las personas se muestran reacias y se advierte un riesgo de violencia; la multa representa un mecanismo para persuadir a la pareja en conflicto por asuntos de convivencia, lo que resulta efectivo porque interrumpe la conducta violenta, sin que se resuelvan las diferencias que motivaron la queja, porque no se modifican o cambian las razones que determinaron las diferencias entre las personas. La intencionalidad de la atención del funcionario claramente se ajusta a las normas jurídicas. 
En este sentido, las posibilidades emergentes del diálogo, como lo anotan Schnitman y Schnitman (2008), impiden que las personas aprendan a reaccionar en forma diferente, dado que no reconocen las posiciones que originaron las diferencias, pero sobre todo la forma como sus emociones contradictorias y reacciones agresivas han surgido, lo cual acentúa el malestar y la tensión. Además, la propuesta de un modelo de atención solidario basado en la identificación de las tareas, metas y desafíos familiares (ICBF, 2008), y la participación de la familia en la generación del cambio tampoco se logra.

En relación con la mediación, Littlejohn y Domenici (2008) explican que se trata de un modelo transformador, centrado en la atención que el mediador presta a las necesidades de los disputantes y a la relación entre las partes, con el propósito de plantear objetivos que transformen la dinámica relacional y creen nuevos niveles de concientización y de reconocimiento entre las partes en conflicto, de modo que sean ellos quienes determinen el curso del proceso. Esta alternativa busca el empoderamiento de las personas, la comprensión de sus propios valores, supuestos, intereses y experiencias relacionadas con el motivo del conflicto.

Es decir, se trata de una intervención dialógica enfocada en el reconocimiento de los intereses personales que justifican el conflicto, la comunicación directa entre las partes y la toma de decisiones respecto a la forma de resolverlo, de forma que los participantes logren reconocer que han construido un conflicto y que también, juntos, pueden encontrar una forma de resolverlo. El propósito es que las partes se hagan consientes de lo que sucede en su forma de interactuar y de las posibilidades de transformar tal interacción.

Vale la pena anotar aquí que, de acuerdo con González-Capitel (citado en Salazar y Vinet, 2001), el proceso de mediación se basa en la voluntariedad y confidencialidad del asunto; la neutralidad del mediador y la flexibilidad o informalidad del procedimiento, lo que hace que sea un sistema más rápido que el sistema legal. En este caso, el papel de un mediador en la resolución del conflicto bus- caría crear una nueva forma de relación entre los integrantes de la familia, cuestión que es vista por los funcionarios de la comisaría como una acción de reparación que es responsabilidad de las entidades prestadoras de servicios en salud.

La violencia familiar no solamente provoca estrés postraumático y ser considerada un asunto de salud mental y física, sino que es también la consecuencia de otras formas de violencia social y el reflejo de las formas de relación sustentadas en una concepción tradicional de roles de género que acentúa en relaciones de dominación. Además, detrás de la queja por conflictos de convivencia familiar existe un malestar psíquico que la persona espera resolver con la intervención profesional, queja a la que no necesariamente se responde; no obstante, las secuelas emocionales de los problemas de convivencia familiar pueden ameritar una intervención psicoterapéutica especializada.

Al respecto, Sánchez (2005) explica que la salud mental de una familia o pareja se mide en la manera como afrontan y resuelven los conflictos. Se hace necesaria la figura del mediador cuando los conflictos no se resuelven entre los involucrados, así sea a través del acuerdo y el encuentro de mecanismos que les permitan mantener una buena relación y manejar el desacuerdo. Agrega que el sistema de la mediación familiar se orienta hacia las consecuencias sociales e individuales de la resolución o no resolución del conflicto. El objetivo es llegar a construir una nueva situación satisfactoria para las partes (solución del conflicto), y que estas aprendan a resolver desacuerdos futuros (prevención); por ello, se debe tener en cuenta que una vez resuelto el conflicto, algunos disputantes no tendrán motivos para volver a encontrarse. En otros casos, sí se seguirán viendo o conviviendo.

Uno de los primeros problemas que se evidencia en la resolución de conflictos dentro de la familia es cuando sus miembros dejan de creer en la posibilidad de la negociación y el diálogo como forma de abordar los problemas; generan alianzas y guerras internas que resultan en divisiones. En otros casos, el fracaso social puede resultar en el abuso de alcohol, drogas, o hasta en la delincuen- 
cia, tal como lo ha señalado Cárdenas (1988). En este mismo sentido, Schiffrin (1996) anota que la confidencialidad, el convencimiento, la buena fe, la confianza y el cambio de mentalidad aseguran acuerdos duraderos, por lo que la mediación puede ser un recurso que ayude a la descongestión de la administración de justicia.

Por otra parte, la orientación familiar y la intervención psicológica corresponderían a etapas que 'reparan' las secuelas físicas y emocionales de la agresión en la familia, siendo de competencia de los profesionales de apoyo psicosocial; además, estas son acompañadas por el sistema de salud, a través de las IPS a las que son remitidos los usuarios, y las EPS a las que acuden. Esta secuencia muestra que las acciones en torno a la promoción de la convivencia familiar tienen un énfasis en la énfasis en la norma jurídica y una posible caución amparada en la ley que provoca que la violencia termine, aunque no implica la solución de los motivos que originaron el conflicto ni tampoco crea nuevas alternativas o modos de relación.

De acuerdo con los funcionarios, el desarrollo de un modelo de atención en el que la promoción de la convivencia fuera central supondría ir más allá de los límites que impone el cargo del funcionario. El funcionario no puede extralimitarse en sus funciones, porque sería sancionado, ni tampoco puede crear una actitud asistencialista entre los usuarios, porque estaría en contra de su misión de promover la construcción de convivencia.

El marco jurídico también representa una lente desde la cual se analizan e interpretan las situaciones que motivan la queja o la consulta, lo que sugiere que las representaciones acerca de la convivencia y la resolución de los conflictos están influenciadas por la necesidad de cumplir con la norma jurídica y asegurar la eficiencia.

Como ya se dijo, la intervención es interpretada como una forma de "mitigación", porque la reparación concibe cómo apoyar a la persona para que recupere el equilibrio emocional inicial, anterior a la situación violenta de la que fue objeto, lo que es de competencia de los funcionarios responsables del apoyo psicosocial. En este sentido, se advierte que los usuarios asumen que la justi- cia equivale a la sanción, pues acuden a la comisaría en la búsqueda de un "castigo menor", que sea efectivo, que no equivalga al encarcelamiento del maltratador (acusado) porque de esta persona depende económicamente, el acusador. La intervención jurídica asegura eficacia inmediata, y quizás esto explica el alto número de asesorías legales que se solicitan.

La normatividad jurídica crea esquemas de interpretación de la realidad que limitan la intervención y la posibilidad de la construcción de convivencia familiar en forma colaborativa (el equipo profesional, la familia y los métodos alternativos de resolución de conflictos), pues no se interviene desde un marco común, en razón a que las representaciones sociales desde las que se imparte justicia están diseñadas para operar y asegurar la eficacia del proceso.

Entonces, varias preguntas surgen en relación con la forma como la interpretación de las leyes actúa como un referente para la intervención profesional, pero también en cuanto a la formación educativa que los profesionales reciben en la actualidad. Por ejemplo: ¿cómo superar los límites disciplinares, de modo que sea posible dialogar y construir conocimiento con otras disciplinas, más allá de la investigación tradicional?, ¿en qué forma redefinir la interpretación que tenemos de las funciones profesionales y, sobre todo, del concepto de competencias profesionales, de modo que se replantee el concepto de trabajo colaborativo y en equipo? Además, ¿cómo promover una mayor participación y empoderamiento de los usuarios que supere el asistencialismo y promueva una concepción renovada de administración de justicia?

Son varias las tareas que enfrenta la formación profesional; sin embargo, es necesario encontrar mecanismos para construir una mentalidad diferente, que enfatice en la construcción conjunta de conocimiento, pero también en el riesgo a "pensar de otro modo", a innovar y transformar en los modos de investigar e intervenir.

La creatividad y la innovación en intervención psicosocial que demandan los nuevos profesionales también pasa por la creación de nuevos escenarios de aprendizaje, nuevos escenarios de formación y 
de intervención con la comunidad, en los que la reflexión ética tenga cabida como un asunto de participación mutua, pues es de esta forma como podrá superarse el asistencialismo de las familias y el intervencionismo de los profesionales.

Otra tarea, aún más importante, es comprender el riesgo que representa una reflexión disciplinar y unilateral, dada la complejidad de los problemas de convivencia familiar; por ello, es necesario estudiar y analizar críticamente la forma como las condiciones históricas, sociales, económicas y políticas han transformado la forma como se construye el conocimiento, vinculando nuevos saberes y experiencias de las entidades públicas y privadas, para aportar a la construcción de conocimiento contextualizado acerca de la realidad de las familias, incluyendo las nuevas formas familiares y las nuevas formas de intervención, como ya se ha dicho.

En este sentido, el marco jurídico colombiano permite la implementación de métodos alternativos de resolución de conflictos, la cual se afianza con la Ley 446 de 1998 para la descongestión, eficiencia y acceso a la justicia, y con el Decreto 1818 de 1998, que promulga el estatuto de los mecanismos alternativos para la resolución de conflictos.

Desde hace más de diez años, este conjunto de leyes sostiene los valores constitucionales de "la convivencia dentro de un marco democrático y participativo", permitiéndoles a los ciudadanos tener dos vías de acceso a la justicia: la judicial o la alternativa. No obstante, la realidad muestra cómo los conflictos se visibilizan e incrementan a diario, y lo hacen de manera paralela al represamiento en los despachos judiciales, mientras fracasan las vías alternativas para su resolución (Sayas, 2009).

Las comisarías de familia parecen ser un escenario privilegiado para la vinculación de las universidades, de modo que la articulación entre la academia y el contexto se haga realidad, y se creen nuevas alternativas para la aplicación de los métodos alternativos de resolución de conflictos y de participación social y familiar. De esta forma, la idea es contribuir al replanteamiento y la innovación en los modelos de promoción de la convi- vencia e intervención de la violencia familiar y los victimarios, a la vez que cuestionar la relación familia, derechos y ciudadanía, siendo conscientes del lugar que tradicionalmente ha tenido la familia como célula básica de la sociedad.

\section{Conclusiones}

Los procesos de atención en la Comisaría de Familia de la comuna 20 en la ciudad de Cali, se ordenan dentro de lo prescrito por la Ley 575 de 2000 sobre asuntos de familia, la Ley 1098 respecto a infracciones de menores, y la Ley 446 de 1998 para la descongestión de la administración de justicia. La intervención se basa en la norma jurídica, por considerar que tiene un efecto inmediato que la hace eficiente. La conciliación representa una alternativa de intervención que reduce o interrumpe el riesgo de una acción violenta, pero no contribuye a la resolución del conflicto ni al alivio del malestar psíquico en la persona violentada. El modelo de atención solidario se sujeta a la normatividad jurídica, lo que resta posibilidades a una intervención constructiva y constructivista. Los modelos de formación educativa de los profesionales en el campo de las ciencias sociales deberían volver su mirada a escenarios reales, en los que el aprendizaje de una nueva epistemología sea posible.

\section{Referencias}

Alcaldía de Santiago de Cali, Red del Buen Trato, y Fundación para la Orientación Familiar (FUNOF). (2004). Memorias del Foro Familia y Convivencia, Cali.

Alcaldía de Santiago de Cali, Secretaría de Desarrollo Territorial y Bienestar Social, Red Municipal del Buen Trato, y Programa Interinstitucional para la Participación y Convivencia Ciudadana EDUPAR (2005). Política pública en convivencia familiar para el municipio de Cali, sancionada mediante el Decreto 0710 de 2005 y el Acuerdo 0231 de 2007, Cali

Alcaldía de Santiago de Cali, y Secretaría de Salud Pública Municipal. (2008). Competencias 
institucionales en la atención a la violencia familiar. Cali: Alcaldía de Santiago de Cali/ Secretaría de Salud Pública Municipal.

Álvarez, M. C., Mora, A., y Román, M. (2012). Modelo de acción social del programa ProniñoColombia: Informe final. Documento Interno. Pontificia Universidad Javeriana, seccional Cali/Universidad Pontificia de Comillas, Madrid/ Fundación para la Orientación Familiar, Cali/ Fundación Telefónica, Cali/ Grupo de Investigación Estudios en Cultura, Niñez y Familia, PUJ, Cali.

Barudy, J. (1998) El dolor invisible de la infancia: Una lectura ecosistémica del maltrato infantil. Barcelona: Paidós.

Bentovim, A. (2000). Sistemas organizados por Traumas: El abuso físico y sexual en las familias. Buenos Aires: Paidós.

Cárdenas, E. J. (1998). La mediación en conflictos familiares. Buenos Aires: Lumen/Hvmanitas.

Comisaría de Familia. (2012). Informes de labores 2007 a 2012. Cali: Comisaría de Familia, sector Ladera/Secretaría de Gobierno/Alcaldía Municipal de Cali.

Constitución Política de Colombia [CPC] (1991). Título II: De los derechos, las garantías y los deberes. Capítulo 2: De los derechos sociales, económicos y culturales, Articulo 42. Julio 7 de 1991.

Corpovisionarios. (2014). Informe final de medición de cultura ciudadana de Cali 2013. Cali: Corpovisionarios.

Eguiluz, L. (2003). Dinámica de la familia. México: Pax México.

Espinosa, M. L. (2007). Perfil de la violencia familiar en Cali: Observatorio de violencia familiar; Informe 2007. Cali: Secretaría de Salud Pública Municipal. Recuperado de http:// www.cali.gov.co/observatorios/publicaciones.php?id=2541
Espinosa, M. L. (2008). Observatorio de violencia familiar: Informe 2008. Cali: Secretaría de Salud Pública Municipal. Recuperado de http://www.cali.gov.co/observatorios/publicaciones.php?id $=2541$

Guío, R. E. (2009). El concepto de familia en la legislación y en la jurisprudencia de la Corte Constitucional Colombiana. Estudiositas, 4(3), 65-81.

Littlejohn, S., y Domenici, K. (2008). Objetivos de la comunicación y métodos de la mediación. En D. F. Schnitman (Comp.), Nuevos paradigmas en la resolución de conflictos, perspectivas y prácticas. Buenos Aires: Granica.

ICBF. (2008). Lineamientos para la atención en comisarías de familia. Bogotá: República de Colombia/Ministerio de la Protección Social/ Instituto Colombiano de Bienestar Familiar.

Instituto Colombiano de Bienestar Familiar (ICBF). (2008). Lineamientos para la atención e inclusión de familias. República de Colombia/ Ministerio de la Protección Social/Instituto Colombiano de Bienestar Familiar.

Mora, A., y Román, M. (2013, agosto 28-30). Convivencia familiar en Cali: Las comisarías de familia, un escenario de formación profesional. Ponencia presentada en el vi Encuentro $\mathrm{Na}$ cional sobre Familias: Retos para la intervención con familias, Universidad del Valle, Cali.

Ortiz, Y., Franco, H., y Campo, D. (2007). Una aproximación a la medición de la violencia familiar en Santiago de Cali, 2003-2005. Recuperado de http://www.revmed.unal.edu. co/revistafm/v55n1/html/v55n1a05.html

Pineda, J., y Otero, P. (2004). Género, violencia familiar e intervención pública en Colombia. Revista de Estudios Sociales, (17), 19-31.

Rentería, E., Lledias, E., y Giraldo, A. L. (2008). Convivencia familiar: Una lectura aproximativa desde elementos de la psicología social. Diversitas: Perspectivas en Psicología, 4(2), 427-441. 
Colombia, Congreso de la República. Ley 446 de 1998. Por la cual se adoptan como legislación permanente algunas normas del Decreto 2651 de 1991, se modifican algunas del Código de Procedimiento Civil, se derogan otras de la Ley 23 de 1991 y del Decreto 2279 de 1989, se modifican y expiden normas del Código Contencioso Administrativo y se distan otras disposiciones sobre la descongestión, eficiencia y acceso a la justicia. Recuperado de http:// www.sic.gov.co/recursos_user/documentos/normatividad/Leyes/1999_Hacia_Atras/ Ley_446_1998.pdf

Colombia, Congreso de la República. Ley 294 de 1996. Por la cual se desarrolla el artículo 42 de la Constitución Política y se dictan normas para prevenir, remediar y sancionar la violencia intrafamiliar (julio 16 de 1996). Recuperado de http://www.elabedul.net/San_Alejo/ Leyes/Leyes_2000/ley_600_2000.php

Colombia, Congreso de la República. Ley 575 de 2000. Por medio de la cual se reforma parcialmente la Ley 294 de 1996 (febrero 9 de 2000). Recuperado de http://www.elabedul.net/San_ Alejo/Leyes/Leyes_2000/ley_600_2000.php

Colombia, Congreso de la República. Ley 1098 de 2006. Por la cual se expide el Código de Infancia y Adolescencia (noviembre 8 de 2006). Recuperado de http://www.elabedul.net/San_ Alejo/Leyes/Leyes_2000/ley_600_2000.php

Colombia, Congreso de la República. Ley 1361 de 2009. Por medio de la cual se crea la Ley Integral de Protección a la Familia (diciembre 3 de 2009). Recuperado de http://www. elabedul.net/San_Alejo/Leyes/Leyes_2000/ ley_600_2000.php
Colombia, Congreso de la República. Decreto 1818 de 1998. Por medio de la cual se expide el Estatuto de los Mecanismos Alternativos de Resolución de Conflictos (24 de septiembre de 2008). Recuperado de http://www.secretariasenado.gov.co/senado/basedoc/decreto_1818_1998.html

Colombia, Congreso de la República. Decreto 2737 de 1989. Por el cual se expide el Código del Menor, se reglamenta la competencia del Defensor de Familia y el tema de las adopciones (filiación adoptiva Art. 112), Deroga la Ley $5^{a}$ de 1975 (Noviembre 27 de 1989). Recuperado de http://www.elabedul.net/San_Alejo/Leyes/Leyes_2000/ley_600_2000.php

Salazar, D., y Vinet, E. (2011). Mediación familiar y violencia de pareja. Revista de Derecho, 24(1), 9-30.

Sayas, R. (2009). Conciliaciones en clave comunicativa o conciliaciones en clave instrumental. Bogotá: Doctrina y Ley.

Schiffrin, A. (1996). La mediación: Aspectos generales. En J. Gottheil y A. Schiffrin (Eds.), Mediación: Una transformación en la cultura. (Comps). Argentina: Paidós-Mediación.

Schnitman, D., y Schnitman, J. (2008) La resolución alternativa de conflictos: Un enfoque generativo. En Nuevos Paradigmas en la Resolución de Conflictos. Buenos Aires: Granica.

Victoria, I., y Bonilla, N. (2008). Imaginarios y marcos valorativos de funcionarios de justicia sobre víctimas de violación sexual en Cali. Pensamiento Psicológico, 4(11), 45-64. 